\title{
Diseño e implementación de una aplicación móvil android para el seguimiento de rutas de transporte urbano en el municipio de Yopal
}

\section{Design and implementation of an android mobile application to follow the routes of urban transport in the area of Yopal}

\section{Policarpo Malabar Galindo Pérez Fundación Universitaria de San Gil, UNISANGIL El Yopal, Colombia}

Correo electronico: policarpogalindo@ unisangil.edu.co

Mónica Andrea Suárez Vargas Autor de correspondencia Fundación Universitaria de San Gil, UNISANGIL El Yopal, Colombia

Correo electronico: msuarez@unisangil.edu.co 


\section{Resumen}

Este documento presenta los resultados del desarrollo de una aplicación móvil Android para la visualización de las rutas de transporte urbano en la ciudad de Yopal, utilizando la metodología XP, que ofrece un marco adaptable a las condiciones del proyecto. El desarrollo de la aplicación se ha realizado con el entorno de desarrollo de Android Studio, Google Maps para Android y Firebase para iniciar sesión con la cuenta de Google y la base de datos. El trabajo está dirigido a los usuarios de la ciudad de Yopal, departamento de Casanare (Colombia), que toman el servicio de transporte urbano con el objetivo de facilitar la información correspondiente a las rutas de los paseos en minibús.

\section{Abstract}

This document presents the results of the development of an Android mobile application for the visualization of the urban transportation routes in Yopal city, making use of the agile XP methodology that offers an adaptable framework to the project conditions. The development of the application has been made with the Android Studio development environment, Google Maps for Android, Firebase to login in with the Google account and database. The application is addressed to the users of Yopal City, Casanare department, who take the urban transportation service, to facilitate the information corresponding to the routes the minibus rides.

\section{Palabras \\ Claves:}

Móvil, Android, rutas, usuario

\section{Keywords:}

Mobile, android, routes, user. 
La globalización tecnológica ha obligado a las empresas a cambiar su forma de realizar los negocios; debido a la gran competencia que esta ha generado en el mercado. Por esta razón, es necesario que las empresas diseñen herramientas para agilizar los procesos que realizan sin perder calidad.

Por otra parte, ahora las personas tienen al alcance dispositivos móviles que soportan la instalación de aplicaciones y datos o conexión a wi-fi para la navegación en Internet, lo que ha modificado sus hábitos de consumo y la forma de adquirir productos [1].

En estas circunstancias, es importante adoptar los cambios que la tecnología ofrece, para tener una mayor interacción con el usuario. En concreto, el uso de aplicaciones móviles facilita la comunicación entre las empresas de transporte urbano y el usuario, por lo que es conveniente mostrar información de manera rápida y sencilla, de modo que el usuario puede acceder a esta desde sus dispositivos electrónicos.

Los usuarios que usan el servicio de transporte urbano desconocen en muchas ocasiones el trayecto y los lugares por los que pasa el microbús, viéndose con frecuencia en la necesidad de preguntarle sobre esto al conductor, y no pocas veces este detiene el vehículo para responder a la inquietud del usuario.

Analizando esta situación, se plantea como solución el desarrollo de Rutas Yopal, una aplicación móvil en Android, que contará con las rutas actualmente disponibles, además de opciones para que el usuario busque la ruta que mejor le sirve para llegar a su destino.

Durante el desarrollo del proyecto se plantean los requisitos funcionales y no funcionales que la aplicación debe cumplir para su correcto funcionamiento. Estos son necesarios para la realización de los diagramas en UML, los cuales permitirán entender la interacción entre esta y el usuario. 
Con los diagramas obtenidos se procede a realizar la codificación haciendo uso del entorno de desarrollo Android Studio. Adicionalmente, se contará con la interacción de la plataforma Firebase para el almacenamiento de los datos a usar en la lista de barrios que contiene la aplicación, y los permisos de Google Maps para que se pueda visualizar el mapa. 
La metodología XP (Extreme Programming) es "un enfoque para el desarrollo de software que utiliza buenas prácticas de desarrollo y las lleva a los extremos. Se basa en valores, principios y prácticas esenciales. Los cuatro valores son la comunicación, la simplicidad, la retroalimentación y la valentía" [2].

La metodología XP propone un lineamiento para garantizar que se hagan las cosas que se consideran más relevantes en el proyecto, dividiéndolo en cuatro fases con el fin de no realizar acciones innecesarias:

- Fase de planeación

- Fase de diseño

- Fase de codificación

- Fase de pruebas

\section{Fase de planeación}

En la fase de planeación se llevaron a cabo reuniones con el jefe operativo de la empresa Coocatrans Ltda., para conocer el número de rutas existentes y de esta forma iniciar la creación de las historias de usuario y definir los requerimientos funcionales y no funcionales para el desarrollo de la aplicación. A continuación, se explica qué significan estas últimas:

Historias de usuario: Constituyen la representación de un requisito que se escribe usando un lenguaje común con el usuario, para determinar las funciones que debe cumplir la aplicación. 
Historia de usuario

\begin{tabular}{|c|c|}
\hline Numero: 01 & Usuario: Cliente \\
\hline Nombre historia: Crear rutas de transporte urbano \\
\hline Prioridad en negocio: Alta & Riesgo de desarrollo: Bajo \\
\hline Puntos estimados: 1 & Iteración asignada: 01 \\
\hline
\end{tabular}

Programador responsable: Policarpo Galindo

Descripción: Trazar las rutas que realizan las busetas en el área urbana de Yopal.

Validación: El usuario puede seleccionar una de las rutas establecidas y ver el trayecto.
Tabla 1.

Crear rutas de transporte.

\begin{tabular}{|c|c|}
\hline \multicolumn{2}{|l|}{ Historia de usuario } \\
\hline Numero: 02 & Usuario: Cliente \\
\hline \multicolumn{2}{|c|}{ Nombre historia: Crear buscador de ruta } \\
\hline Prioridad en negocio: Alta & Riesgo de desarrollo: Bajo \\
\hline Puntos estimados: 1 & Iteración asignada: 02 \\
\hline \multicolumn{2}{|c|}{ Programador responsable: Policarpo Galindo } \\
\hline \multicolumn{2}{|c|}{$\begin{array}{l}\text { Descripción: Crear una lista de barrios por los cuales pasa la } \\
\text { buseta, para poder consultar escribiendo el nombre del barrio. }\end{array}$} \\
\hline \multicolumn{2}{|c|}{$\begin{array}{l}\text { Validación: El usuario obtendrá una respuesta luego de escribir el } \\
\text { nombre de un barrio. }\end{array}$} \\
\hline
\end{tabular}

Tabla 2.

Crear bucador de rutas

\section{Requerimientos funcionales: Indican cómo debe responder la aplicación cuando el usuario interactúa con ella.}


Tabla 3.

Autenticación de usuario Localización

Tabla 5. Búsqueda de barrios

\begin{tabular}{|c|l|l|l|}
\hline Código & RF01 & Prioridad & E-Esencial \\
\hline \multicolumn{2}{|c|}{ Título } & \multicolumn{2}{|c|}{ Autenticación de usuario } \\
\hline
\end{tabular}

Descripción:

El usuario deberá autenticarse utilizando una cuenta activa de Google para acceder a la aplicación. Una vez autenticado no tendrá que realizar este proceso hasta que seleccione la opción "cerrar sesión".

Si el usuario no se ha autenticado, tendrá la opción de añadir una cuenta para que seleccione una existente o cree una nueva cuenta en Google.

\begin{tabular}{|c|l|c|l|}
\hline Código & RF02 & Prioridad & E-Esencial \\
\hline \multicolumn{2}{|c|}{ Lótulo } & \multicolumn{2}{|c|}{ Localización } \\
\hline \multicolumn{2}{|c|}{ Tín } \\
\hline
\end{tabular}

Descripción:

$\mathrm{Al}$ ingresar el usuario a la aplicación, está lo georreferenciará en el punto de localización en el que se encuentre respecto a las coordenadas del mapa de Yopal.

\begin{tabular}{|c|l|l|l|}
\hline Código & RF03 & Prioridad & E-Esencia \\
\hline \multicolumn{2}{|c|}{ Título } & \multicolumn{3}{|c|}{ Búsqueda de barrio } \\
\hline
\end{tabular}

Descripción:

El usuario tendrá la opción de hacer una búsqueda ingresando el nombre del barrio y la aplicación mostrara las rutas que pasan por este barrio. Esta información se mostrará en una lista. 


\begin{tabular}{|c|c|c|c|}
\hline Código & RF04 & Prioridad & E-Esencial \\
\hline \multicolumn{2}{|c|}{ Título } & \multicolumn{2}{|c|}{ Trayecto } \\
\hline \multicolumn{4}{|c|}{ Descripción: } \\
\hline \multicolumn{4}{|c|}{$\begin{array}{l}\text { El usuario seleccionara una de las opciones que se encuentra en el } \\
\text { menú (ruta } 1, \ldots \text { ruta } 13 \text { ) y la aplicación le mostrará en un mapa el } \\
\text { trayecto que sigue la buseta. }\end{array}$} \\
\hline
\end{tabular}

Tabla 6.

trayecto

\begin{tabular}{|c|c|c|c|}
\hline Código & RF05 & Prioridad & E-Esencial \\
\hline \multicolumn{2}{|c|}{ Título } & \multicolumn{2}{|c|}{ Cerrar sesión } \\
\hline \multicolumn{4}{|c|}{ Descripción: } \\
\hline \multicolumn{4}{|c|}{$\begin{array}{l}\text { El usuario podrá salir de la aplicación seleccionando la opción } \\
\text { "Cerrar sesión" que se encuentra en el menú. }\end{array}$} \\
\hline
\end{tabular}

Tabla 6.

Cerrar sesión

Requerimientos no funcionales: Son necesarios para el correcto funcionamiento de la aplicación. 


\begin{tabular}{|c|l|l|l|}
\hline Código & RF01 & Prioridad & E-Esencial \\
\hline \multicolumn{2}{|c|}{ Título } & \multicolumn{3}{|c|}{ Facilidad de uso } \\
\hline
\end{tabular}

Descripción:

Tabla 8.

Facilidad de uso

ELa aplicación debe ser fácil de usar, para todo tipo de usuarios (expertos y no expertos).

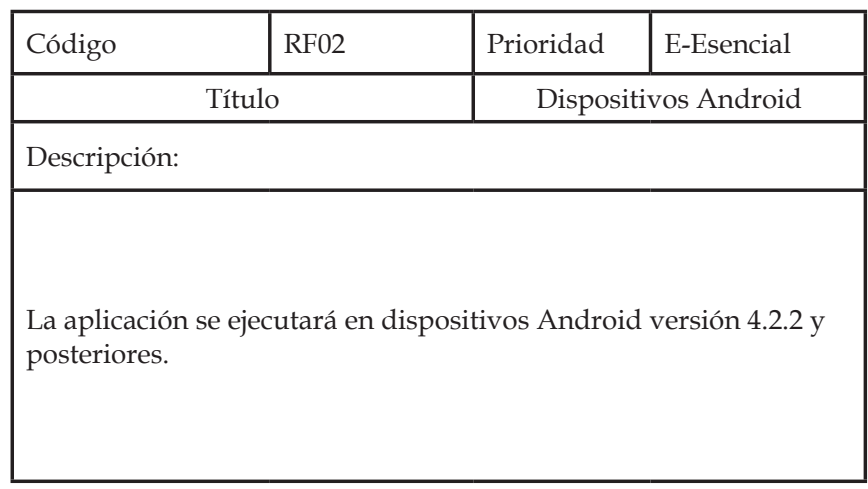

Tarea de ingeniería

Número tarea: 2

Número historia: 1. Crear rutas de transporte urbano

Nombre tarea: Visualizar el trayecto de las rutas

\begin{tabular}{l|l} 
Nombre tarea: Visualizar el trayecto de las rutas \\
\hline
\end{tabular}

Fecha de inicio: Agosto 16 de 2016 Fecha Fin: Octubre 15 de 2016

Programador responsable: Policarpo Malabar Galindo Pérez

Descripción: Desarrollar y programar la interfaz para que los usuarios puedan visualizar el trayecto que realiza la buseta, a través de la aplicación.

Validación: El usuario obtendrá una respuesta luego de escribir el nombre de un barrio. 


\begin{tabular}{|c|c|c|c|}
\hline Código & RF03 & Prioridad & E-Esencial \\
\hline \multicolumn{2}{|c|}{ Título } & \multicolumn{2}{|c|}{ Base de datos Firebase } \\
\hline \multicolumn{4}{|c|}{ Descripción: } \\
\hline \multicolumn{4}{|c|}{$\begin{array}{l}\text { Se hace uso de la plataforma Firebase para almacenar la } \\
\text { información de los barrios. }\end{array}$} \\
\hline
\end{tabular}

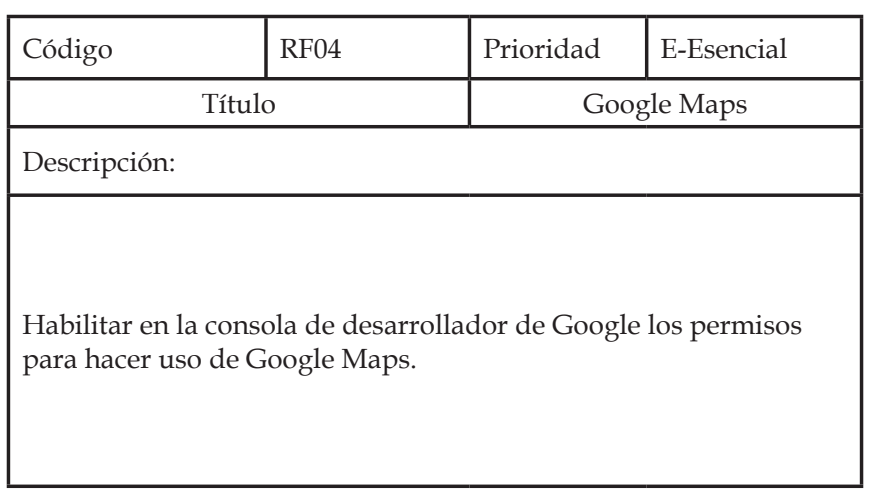

Tabla 11.

Google Maps

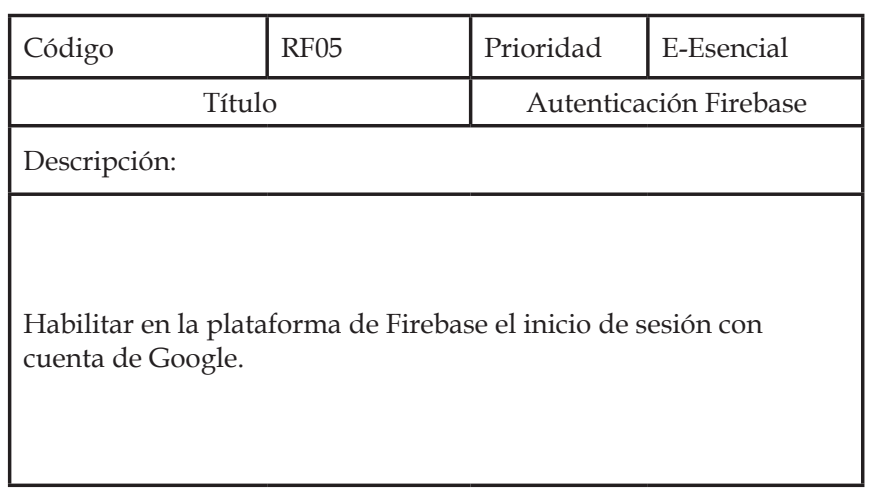

Tabla 12.

Autenticación Firebase 


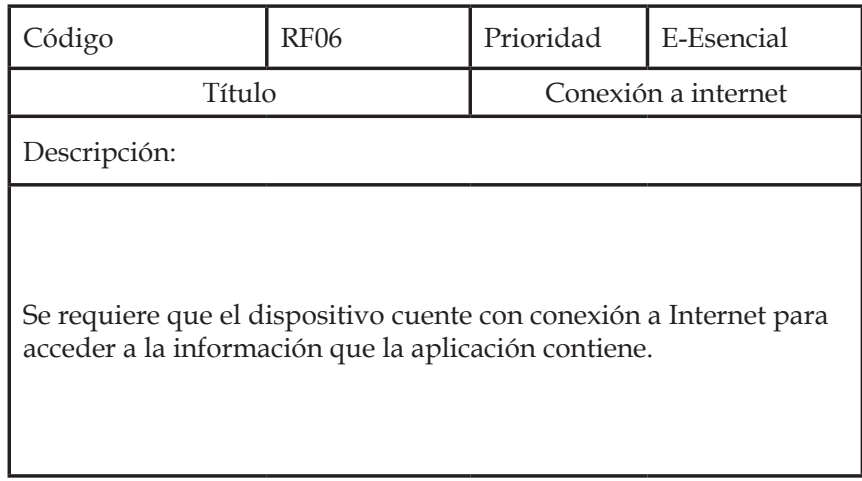

\section{Tareas de ingeniería}

Tabla 14.

Diseño de diagramas UML

Tarea de ingeniería

\begin{tabular}{|c|c|}
\hline Número tarea: 1 & Número historia: \\
\hline \multicolumn{2}{|c|}{ Nombre tarea: Diseño de diagramas UML } \\
\hline Tipo de tarea: Diseño & Puntos estimados: \\
\hline Fecha de inicio: Julio 29 de 2016 & Fecha Fin: Agosto 15 de 2016 \\
\hline \multicolumn{2}{|c|}{ Programador responsable: Policarpo Malabar Galindo Pérez } \\
\hline \multicolumn{2}{|c|}{$\begin{array}{l}\text { Descripción: Realizar diagramas UML (Diagrama de casos de } \\
\text { uso, diagrama de actividades y diagrama de secuencias), para } \\
\text { comprender la interacción entre el usuario y el sistema. }\end{array}$} \\
\hline & \\
\hline
\end{tabular}




\begin{tabular}{|c|c|}
\hline \multicolumn{2}{|l|}{ Tarea de ingeniería } \\
\hline Número tarea: 3 & $\begin{array}{l}\text { Número historia: } 2 \text {. Crear } \\
\text { buscador de ruta }\end{array}$ \\
\hline \multicolumn{2}{|l|}{ Nombre tarea: Buscar y mostrar barrios } \\
\hline Tipo de tarea: Desarrollo & Puntos estimados: \\
\hline $\begin{array}{l}\text { Fecha de inicio: Noviembre } 15 \text { de } \\
2016\end{array}$ & $\begin{array}{l}\text { Fecha Fin: Diciembre } 14 \text { de } \\
\qquad 2016\end{array}$ \\
\hline \multicolumn{2}{|c|}{ Programador responsable: Policarpo Malabar Galindo Pérez } \\
\hline \multicolumn{2}{|c|}{$\begin{array}{l}\text { Descripción: Se creará una lista de barrios, para que los usuarios } \\
\text { puedan consultar la ruta que quieren, escribiendo el nombre del } \\
\text { barrio. }\end{array}$} \\
\hline $\begin{array}{l}\text { Validación: El usuario obtendrá } \\
\text { nombre de un barrio. }\end{array}$ & puesta luego de escrib \\
\hline
\end{tabular}

\begin{tabular}{|c|c|}
\hline \multicolumn{2}{|l|}{ Tarea de ingeniería } \\
\hline Número tarea: 4 & Número historia: \\
\hline \multicolumn{2}{|l|}{ Nombre tarea: Inicio de sesión } \\
\hline Tipo de tarea: Desarrollo & Puntos estimados: \\
\hline $\begin{array}{l}\text { Fecha de inicio: Diciembre } 14 \text { de } \\
2016\end{array}$ & Fecha Fin: Enero 16 de 2017 \\
\hline \multicolumn{2}{|c|}{ Programador responsable: Policarpo Malabar Galindo Pérez } \\
\hline \multicolumn{2}{|c|}{$\begin{array}{l}\text { Descripción: Desarrollar y programar la interfaz de inicio } \\
\text { de sesión, para que los usuarios ingreses los datos (correo y } \\
\text { contraseña), que serán validados por el sistema, para realizar el } \\
\text { registro en la aplicación. Una vez validado el usuario ingresara a } \\
\text { la actividad principal de la aplicación. }\end{array}$} \\
\hline \multicolumn{2}{|c|}{$\begin{array}{l}\text { Validación: El usuario obtendrá una respuesta luego de escribir el } \\
\text { nombre de un barrio. }\end{array}$} \\
\hline
\end{tabular}

Tabla 15.

Buscar y mostrar barrios

\section{Tabla 16.}

Inicio de sesión 


\section{Fase de diseño}

En la fase de diseño se realizan los diagramas UML (Lenguaje Unificado de Modelado), a fin de tener una perspectiva de lo que hace el sistema al interactuar con el usuario.

Casos de uso: Se establecen los actores que van a intervenir en el desarrollo de la aplicación, teniendo en cuenta los requisitos planteados anteriormente, para dar cumplimiento a la tarea que cada uno debe cumplir en la la realización del proyecto [3].

Figura 1.

Caso de Uso - Inicio

de sesión

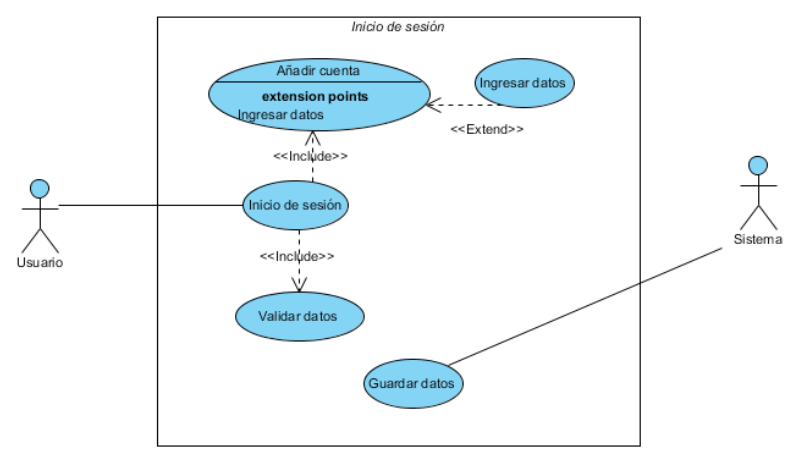




\begin{tabular}{|c|l|}
\hline Nombre del caso de uso: & Inicio de sesión \\
\hline Actor: & Usuario \\
\hline Propósito: & Ingresar a la aplicación \\
\hline Resumen: & $\begin{array}{l}\text { El usuario ingresa sus datos de } \\
\text { su cuenta en Google (correo y } \\
\text { contraseña). }\end{array}$ \\
\hline Pre-condición & $\begin{array}{l}\text { El usuario debe tener Internet } \\
\text { (datos o wi-fi). }\end{array}$ \\
\hline Usuario & Sistema \\
\hline 1. El usuario ingresa a la aplicación & $\begin{array}{l}\text { 2. El sistema muestra botón de } \\
\text { iniciar sesión }\end{array}$ \\
\hline $\begin{array}{l}\text { 3. El usuario selecciona botón e } \\
\text { ingresa los datos correspondientes }\end{array}$ & $\begin{array}{l}\text { 4. El sistema valida la información } \\
\text { suministrada. Si los datos son } \\
\text { válidos los envía a la actividad } \\
\text { principal; de lo contrario, envía un } \\
\text { mensaje informando que no se ha } \\
\text { podido iniciar sesión. }\end{array}$ \\
\hline & 5. El sitema guarda la información. \\
\hline
\end{tabular}

\section{Tabla 17.}

Caso de uso - inicio de sesión

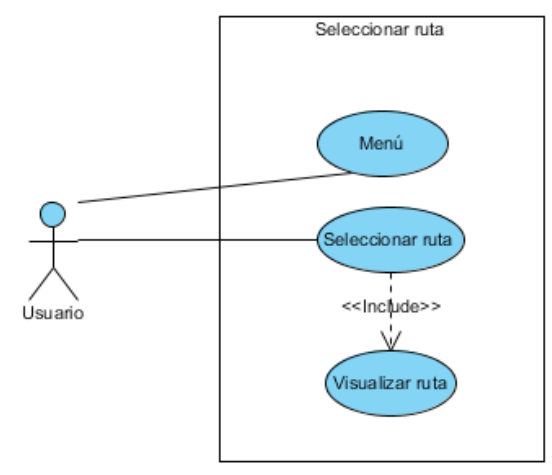

Figura 2.

Caso de Uso -

Seleccionar ruta 


\begin{tabular}{|c|c|c|}
\hline & Nombre del caso de uso: & Seleccionar ruta \\
\hline & Actor: & Usuario \\
\hline & Propósito: & Seleccionar una opción del menú \\
\hline & Resumen: & $\begin{array}{l}\text { Cuando el usuario haya iniciado } \\
\text { sesión, podrá seleccionar una ruta y } \\
\text { ver el trayecto trazado en el mapa. }\end{array}$ \\
\hline & Pre-condición & $\begin{array}{l}\text { El usuario debe tener internet } \\
\text { (datos o wi-fi). }\end{array}$ \\
\hline & $\begin{array}{l}\text { El usuario debe haber iniciado } \\
\text { sesión. }\end{array}$ & Sistema \\
\hline \multirow{3}{*}{$\begin{array}{r}\text { Tabla } 18 . \\
\text { Caso de uso - } \\
\text { seleccionar ruta }\end{array}$} & Usuario & Sistema \\
\hline & 1. El usuario inicia sesión & 2. El sistema muestra el mapa \\
\hline & 3. El usuario selecciona una ruta & 4. El sistema muestra el trayecto \\
\hline
\end{tabular}

Figura 3.

Caso de Uso -

Buscar ruta

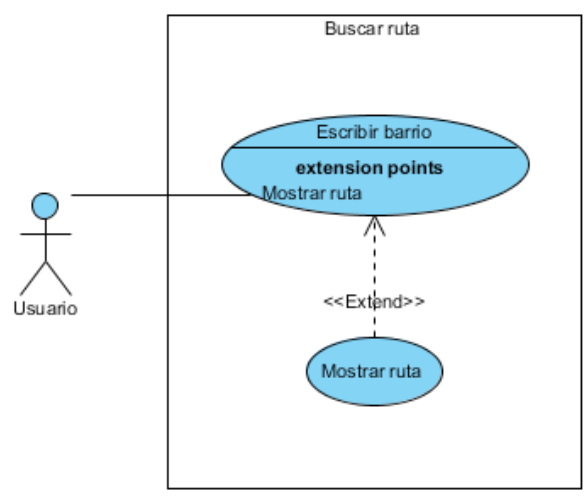




\begin{tabular}{|c|l|}
\hline Nombre del caso de uso: & Buscar ruta \\
\hline Actor: & Usuario \\
\hline Propósito: & $\begin{array}{l}\text { Filtrar lista de acuerdo con el barrio } \\
\text { que se escriba }\end{array}$ \\
\hline Resumen: & $\begin{array}{l}\text { Cuando el usuario seleccione la } \\
\text { opción de barrios, se mostrará la } \\
\text { lista de barrios y contará con la } \\
\text { opción de búsqueda para filtrar los } \\
\text { resultados. }\end{array}$ \\
\hline Pre-condición & $\begin{array}{l}\text { El usuario debe tener Internet } \\
\text { (datos o wi-fi). }\end{array}$ \\
\hline El usuario debe haber iniciado & Sesión. \\
\hline Usuario & Sistema \\
\hline $\begin{array}{l}\text { 1. El usuario selecciona opción } \\
\text { barrios }\end{array}$ & $\begin{array}{l}\text { 2. El sistema muestra lista de } \\
\text { barrios }\end{array}$ \\
\hline $\begin{array}{l}\text { 3. El usuario escribe el nombre de } \\
\text { un barrio }\end{array}$ & $\begin{array}{l}\text { 4. El sistema filtra la lista y muestra } \\
\text { los resultados que corresponden } \\
\text { con la búsqueda. }\end{array}$ \\
\hline
\end{tabular}

Tabla 19.

Caso de uso - buscar ruta

Diagramas de secuencias: Muestra una interacción que representa la secuencia de mensajes entre instancias de clases, componentes, subsistemas o actores. El tiempo fluye por el diagrama y muestra el flujo de control de un participante a otro. Se utilizan diagramas de secuencia para visualizar instancias y eventos en lugar de clases y métodos. En el diagrama, puede aparecer más de una instancia del mismo tipo. También puede haber más de una ocurrencia del mismo mensaje. [4]. 
Figura 4.

Diagrama de secuencia - Inicio de sesión

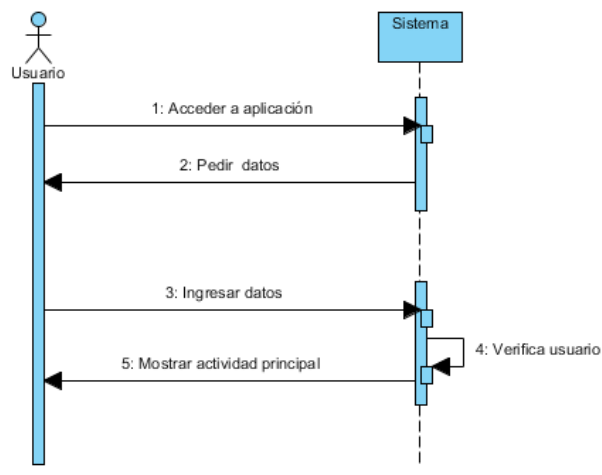

Figura 5.

Diagrama de secuencia Seleccionar ruta

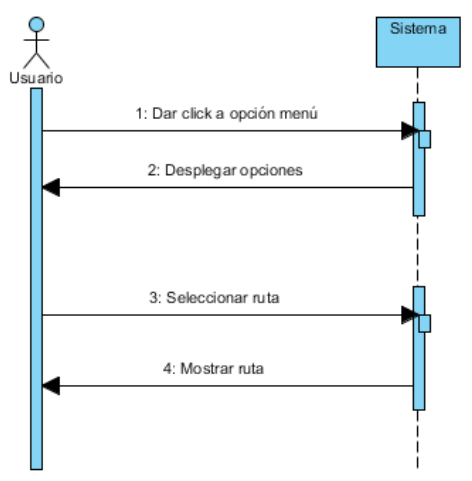

Figura 6. Diagrama de secuencia-Buscar ruta

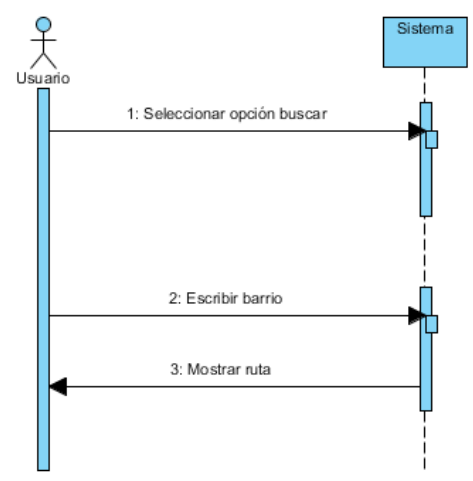


Diagramas de actividades: Muestra el proceso que realiza el programa a través de una serie de acciones entre el usuario y el sistema al momento de ejecutarlo [5].

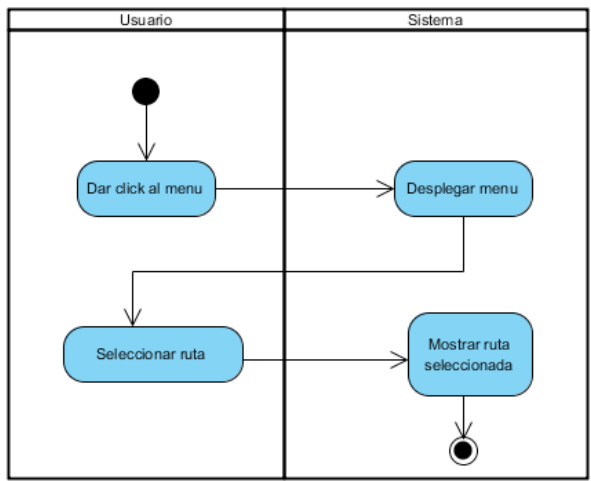

Figura 7.

Diagrama de actividades - Inicio de sesión

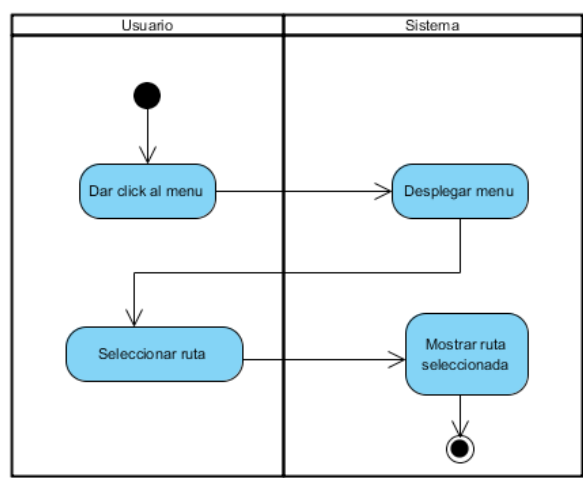

Figura 8.

Diagrama de actividades Seleccionar ruta 


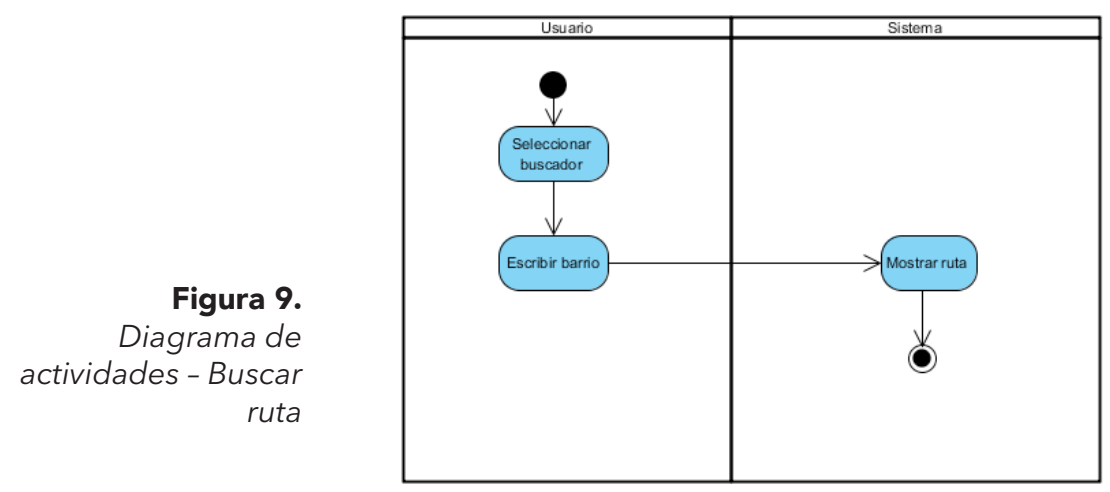

\section{Fase de codificación}

Se mostrará una parte del código y la interfaz gráfica que se utilizó para llevar a cabo el proyecto.

Login: Se habilita en la plataforma de Firebase el uso de la cuenta de Google para el inicio de sesión (ver Fig. 10).

Figura 10.

Método de inicio de

sesión

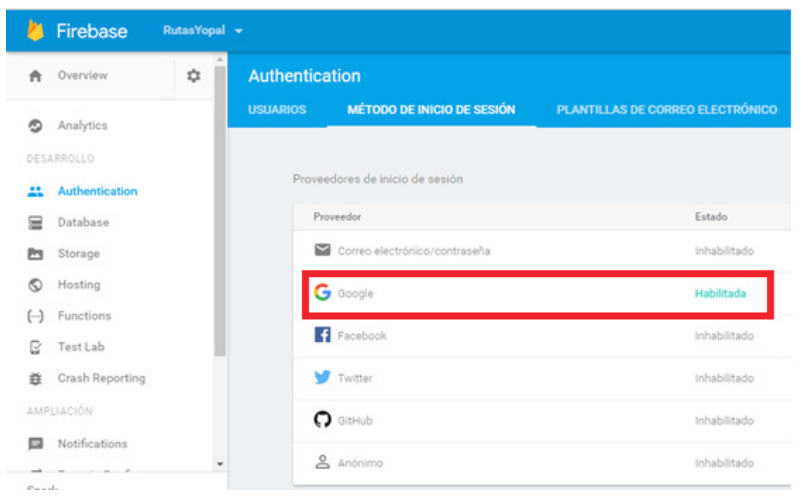


En la Fig. 11, por otro lado, se enseña parte del código que se usó para llevar a cabo la comprobación y el registro de la cuenta de Google en la aplicación.

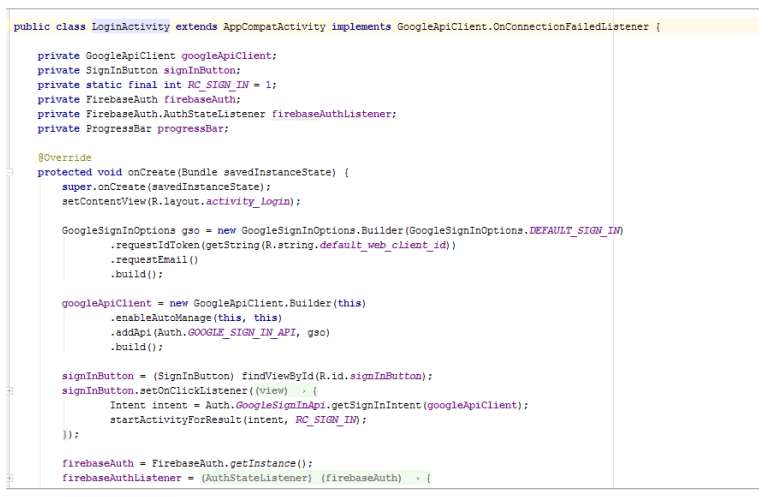

Figura 11.

Código para

LoginActivity

La Fig. 12 constituye la interfaz necesaria para que el usuario seleccione una cuenta o añada otra para ingresar a la información de la aplicación.

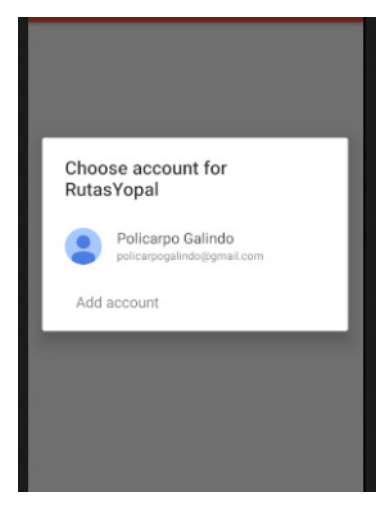

\section{Figura 11.}

Interfaz de inicio de sesión

Menú: Para la navegación en la aplicación se ha usado un NavDrawer, que permite tener un menú desplegable, para que el usuario seleccione una de las opciones. 
En la Fig. 13 se enseña parte del código que se usó para crear el menú en la aplicación.

Figura 13.

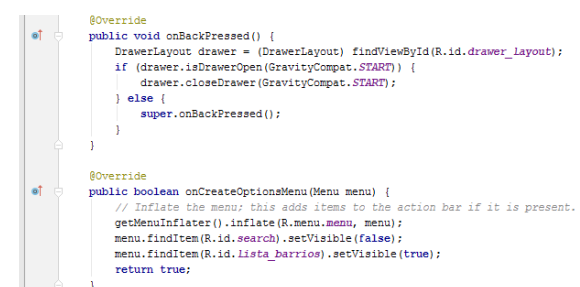

Código para menú

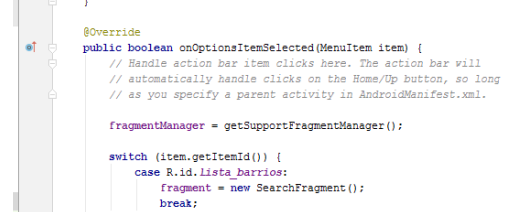

La Fig. 14 reproduce la interfaz para que el usuario seleccione una de las opciones de ruta para visualizarla en el mapa

Figura 14.

Interfaz del menú

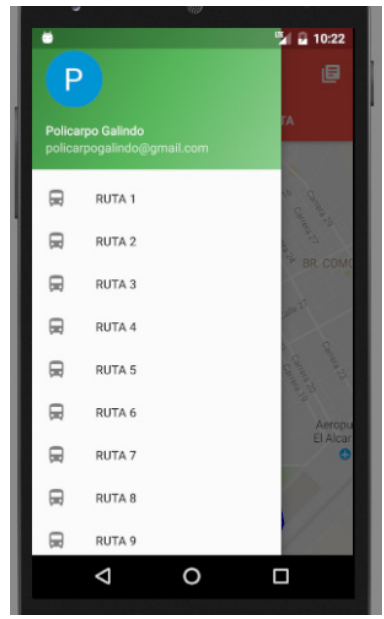


Google Maps: En la consola se debe registrar el desarrollador de Google, para habilitar la Api de Google Maps y tener el permiso de usarla en la aplicación móvil (ver Fig. 15).

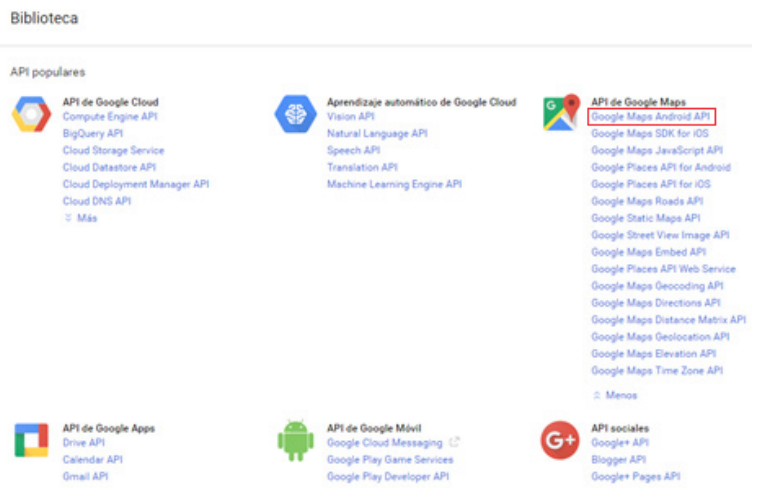

Figura 15.

Habilitar Google

Maps

En la Fig. 16 se enseña parte del código que se usó para implementar el método OnMapReadyCallback, para que la poli línea se pueda ver en el mapa al momento de ejecutar la aplicación.

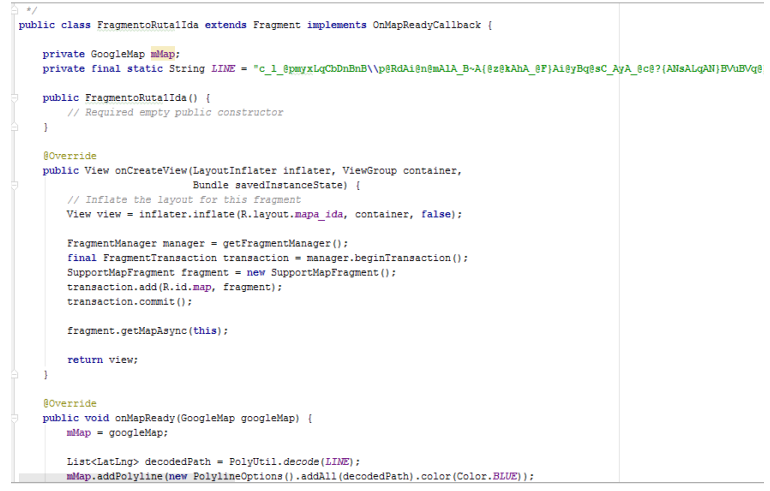

Figura 16

Código de fragment 
La Fig. 17 es la interfaz de la aplicación para visualizar el trayecto de la ruta en el mapa.

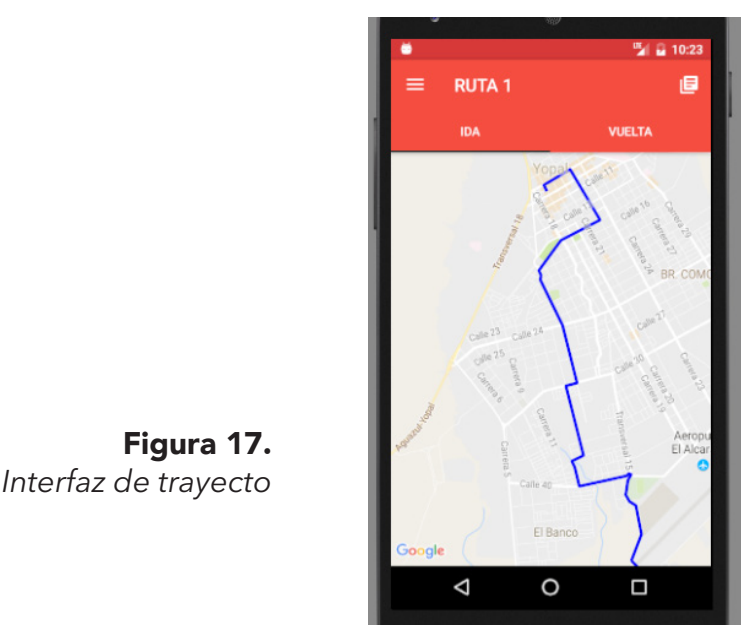

RecyclerView: Esta aplicación se usa para mostrar la información de los barrios en una lista, también se usará para que al momento de hacer la búsqueda se filtre la información y esta se muestra de manera ordenada en la lista.

Figura 18. RecyclerView [6]

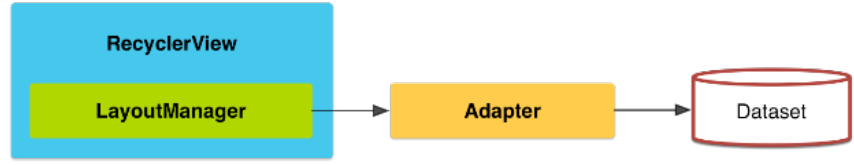


En la Fig. 19 se hace referencia a la conexión con la base de datos en Firebase para que se muestre la información almacenada.

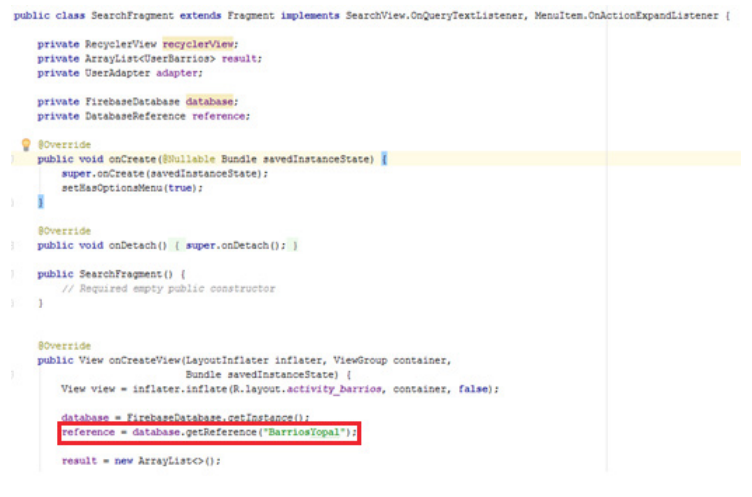

Figura 19.

Código conexión

con Firebase

En la Fig. 20 se enseña parte del código del adaptador del RecyclerView.

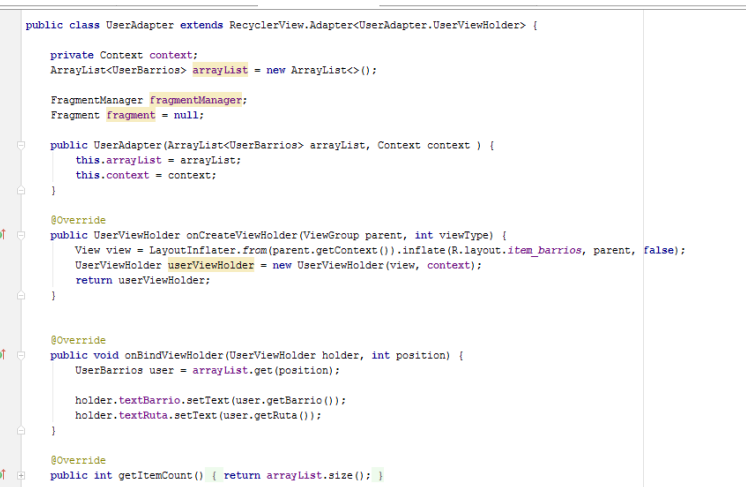

Figura 20.

Código UserAdapter 
La Fig. 21 enseña parte del código que contiene las variables usadas en el recyclerview, estas deben coincidir con las que se encuentran en la base de datos para evitar conflictos.

Figura 21. Código UserBarrios

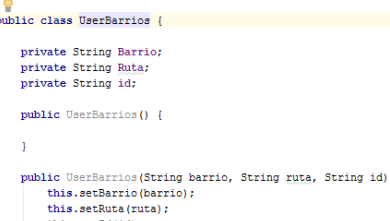

La Fig. 22 es el formato JSON, para la creación de la base de datos en Firebase.

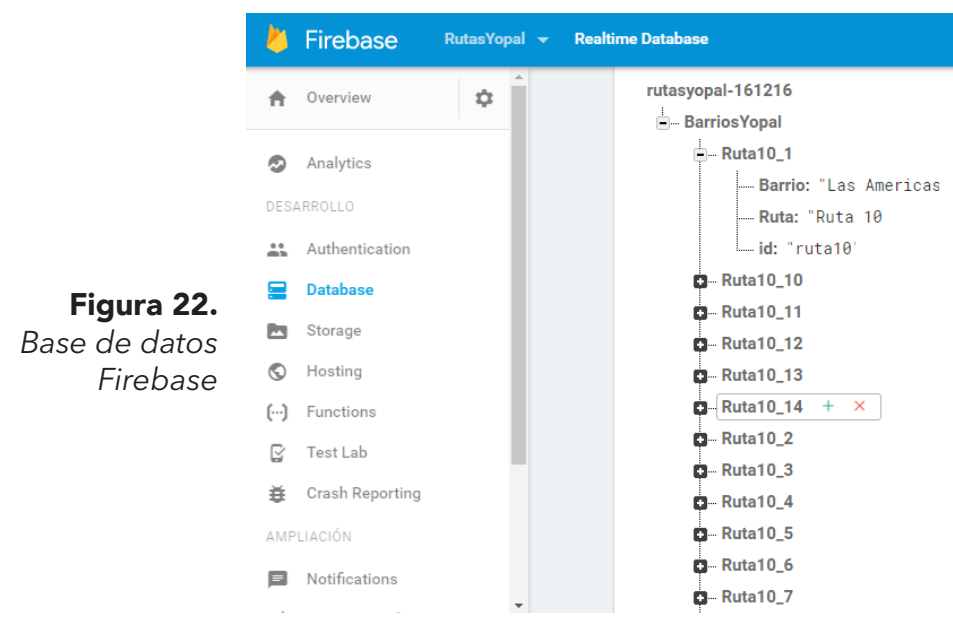


La Fig. 23 representa el ejemplo para habilitar el permiso de lectura, con el fin de que la aplicación acceda a la información de los barrios, que se encuentra almacenada en la base de datos.

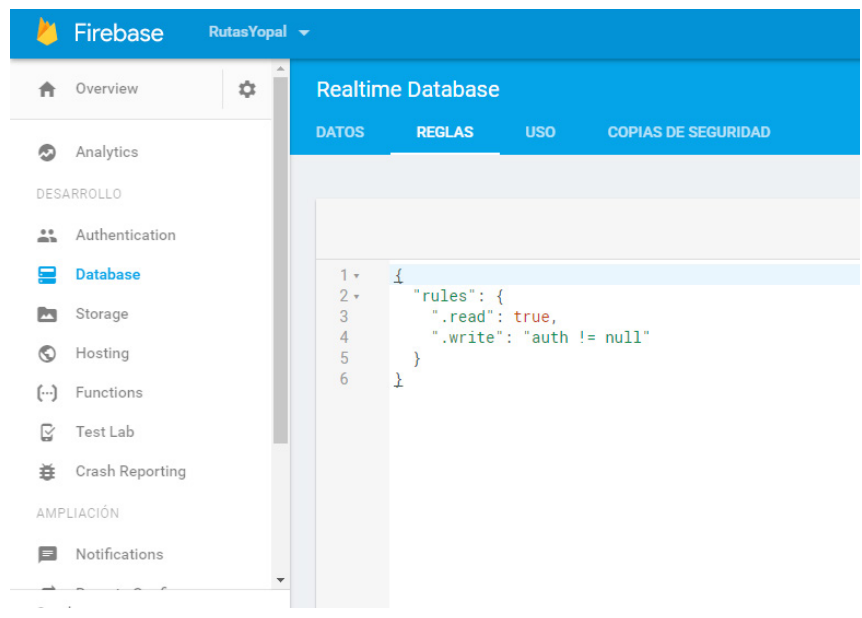

Figura 23.

Habilitar permiso de lectura

En la Fig. 24 se enseña la lista de barrios, y en la 25 se realiza el filtrado escribiendo el nombre del barrio.

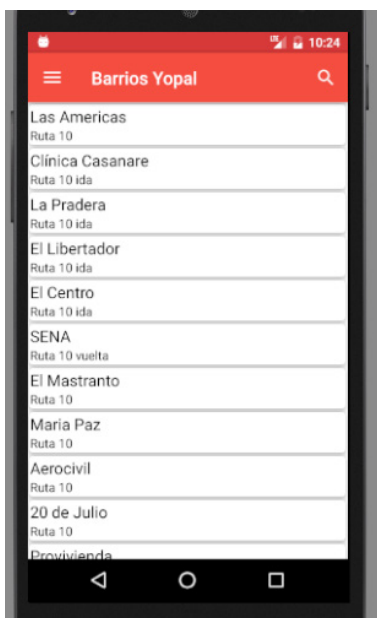

Figura 24.

Interfaz de lista de barrios 
Figura 25.

Interfaz de búsqueda de barrios

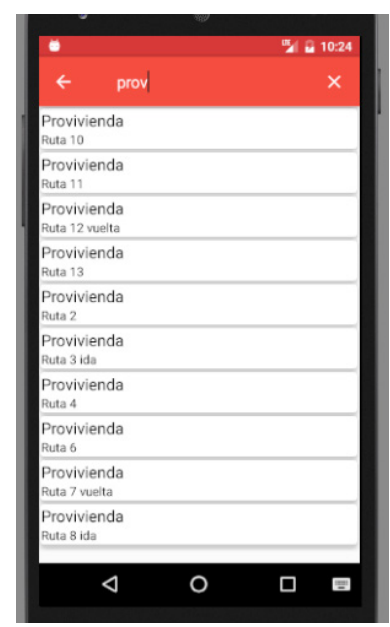

\section{Fase de pruebas}

Se realizan cuando se han completado las etapas anteriores y se inicia el correcto funcionamiento del sistema desarrollado. Esta etapa es crucial en el proceso porque permite detectar errores y corregirlos.

Para realizar las pruebas unitarias se utilizó AndroidTest, que viene incluido en Androd Studio . Este test se puede ejecutar en el JVM local o mediante una prueba instrumentada que se ejecute en un dispositivo

Pruebas unitarias: Las pruebas realizadas se hicieron en unas secciones del código para verificar el correcto funcionamiento de los permisos que se habilitan en Android y verificar la conexión que se tiene (wi-fi o datos). Estos permisos son el de INTERNET, ACCESS_FIN_LOCATION Y ACCES_NETWORK_STATE. 


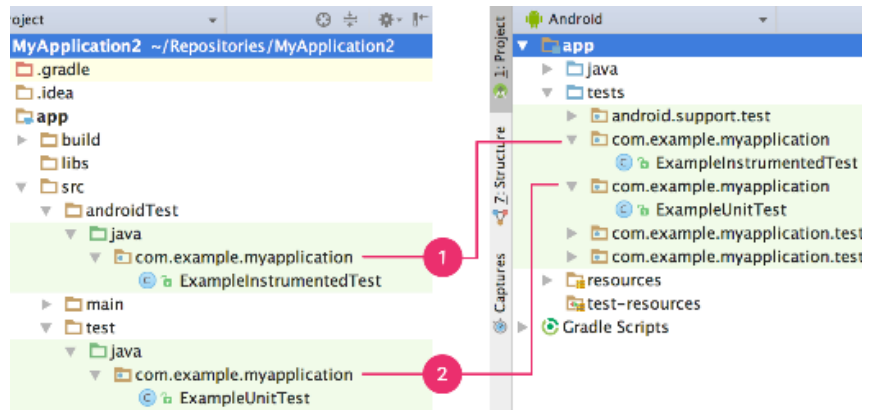

Figura 26.

Las pruebas

instrumentadas (1)

de tu proyecto $y(2)$

las pruebas JVM

locales [7]

En la Fig. 27 se enseña el código utilizado para llevar a cabo la prueba de conectividad, y en la Fig. 28 se presenta el resultado obtenido.

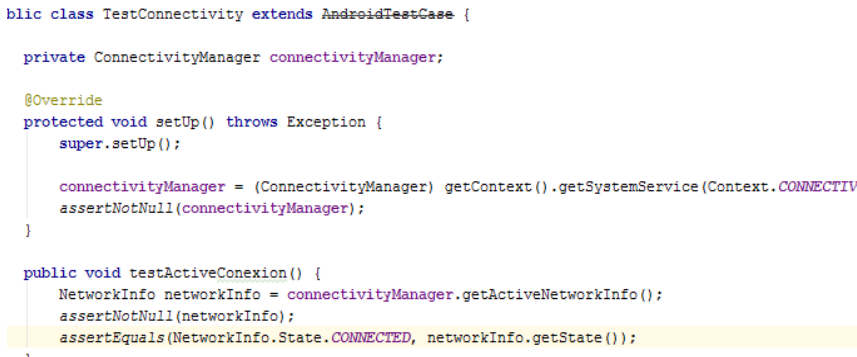

Vol 5 No2 / Julio a Diciembre 2017 Diseño e implementación de una aplicación móvil android para el seguimiento de rutas de transporte urbano en el municipio de Yopal

\section{Figura 27.}

Código prueba de conectividad 

Class com.galindo. policarpo.rutasyopal.test. TestConnectivity
all > com.galindo.policarpo.rutasyopal.test > TestConnectivity

$\begin{array}{ccc}2 & 0 & 0.001 \mathrm{~s} \\ \text { tests } & \text { failures } & \begin{array}{r}100 \% \\ \text { duccessfion }\end{array}\end{array}$

Figura 28.

Resultados prueba de conectividad

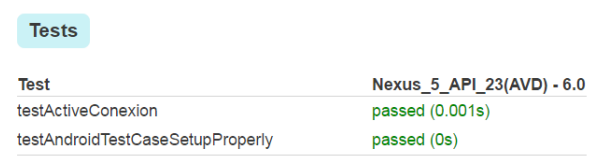

La Fig. 29 reproduce el código utilizado para llevar a cabo la prueba de los permisos que requiere la aplicación y la 30 registra un error en AccesFineLocation, debido a que no estaba habilitado el GPS en el celular.

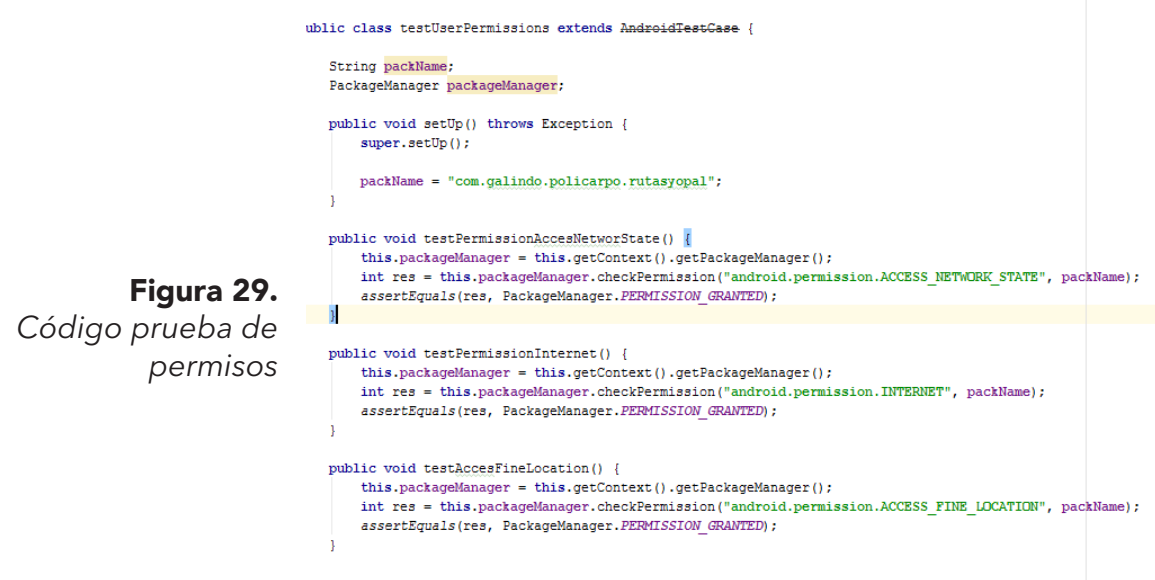


Class com.galindo.policarpo.rutasyopal.test.testUserF

all > com.galindo.policarpo.rutasyopal.test > testUserPermissions

\begin{tabular}{ccc|}
\hline 4 & 1 & $0.006 \mathrm{~s}$ \\
tests & failures & duration \\
\hline
\end{tabular}

\section{$75 \%$}

successful

\begin{tabular}{ll}
\hline Failed tests Tests & \\
Test & Nexus_5_API_23(AVD) -6.0 \\
\hline testAccesFineLocation & failed (0.005s) \\
testAndroidTestCaseSetupProperly & passed (Os) \\
testPermissionAccesNetworState & passed (0.001s) \\
testPermissioninternet & passed (0s)
\end{tabular}

Figura 30.

Resultados prueba

de permisos

En la Fig. 30 se registra la prueba de conexión entre Firebase y Android Studio, lo que generó un error que no permitía que la información de la base de datos alojada en Firebase se visualizara en la pantalla del celular. Una vez realizadas las correcciones necesarias. el sistema no genero más mensajes de error.

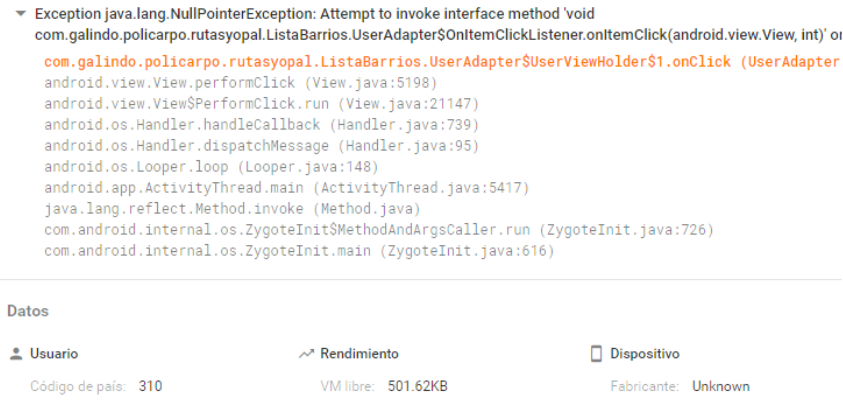

Figura 31.

Prueba UserAdapter 
Pruebas de aceptación: Se realizan con el usuario para verificar el funcionamiento de la aplicación y que este dé la aprobación.

Tabla 20.
Prueba de aceptación 01

\begin{tabular}{|c|c|c|}
\hline \multirow{5}{*}{$\begin{array}{r}\text { Tabla } 20 . \\
\text { Prueba de aceptación } 01\end{array}$} & PRUEBA DE ACEPTACIÓN 01 & Seleccionar ruta \\
\hline & Descripción & $\begin{array}{l}\text { Comprobar que los usuarios } \\
\text { puedan registrarse con su cuenta } \\
\text { de Google, en caso de no tenerla } \\
\text { contar con la opción añadir cuenta } \\
\text { y finalmente acceder a la aplicación. }\end{array}$ \\
\hline & Especificaciones de entrada & $\begin{array}{l}\text { Cuenta de Google (correo, } \\
\text { contraseña). }\end{array}$ \\
\hline & Criterios de aceptación & $\begin{array}{l}\text { Que el usuario acceda a la } \\
\text { aplicación haciendo uso de su } \\
\text { cuenta de Google. }\end{array}$ \\
\hline & \multicolumn{2}{|l|}{ Si cumple } \\
\hline \multirow{5}{*}{$\begin{array}{r}\text { Tabla } 21 . \\
\text { Prueba de aceptación } 02\end{array}$} & PRUEBA DE ACEPTACIÓN 02 & Seleccionar ruta \\
\hline & Descripción & $\begin{array}{l}\text { Comprobar que las rutas de las } \\
\text { busetas se puedan visualizar en el } \\
\text { mapa. }\end{array}$ \\
\hline & Especificaciones de entrada & Estar logueado en la aplicación. \\
\hline & Criterios de aceptación & $\begin{array}{l}\text { Que el usuario pueda seleccionar } \\
\text { la ruta que quiere ver y que esta se } \\
\text { muestre en el mapa. }\end{array}$ \\
\hline & \multicolumn{2}{|l|}{ Si cumple } \\
\hline
\end{tabular}

Tabla 21. 


\begin{tabular}{|c|l|}
\hline PRUEBA DE ACEPTACIÓN 03 & Seleccionar ruta \\
\hline Descripción & $\begin{array}{l}\text { Comprobar que se visualice la lista } \\
\text { de barrios por los que pasan las } \\
\text { busetas y realizar la búsqueda por } \\
\text { barrio. }\end{array}$ \\
\hline Especificaciones de entrada & Estar logueado en la aplicación. \\
\hline Criterios de aceptación & $\begin{array}{l}\text { que el usuario tenga acceso a la lista } \\
\text { de barrios y que el resultado de la } \\
\text { búsqueda se corresponda con el } \\
\text { barrio que se escribe. }\end{array}$ \\
\hline Si cumple & \\
\hline
\end{tabular}

Tabla 22.

Prueba de aceptación 03

\begin{tabular}{|c|l|}
\hline PRUEBA DE ACEPTACIÓN 04 & Seleccionar ruta \\
\hline Descripción & $\begin{array}{l}\text { Comprobar que el usuario pueda } \\
\text { salir de la aplicación. }\end{array}$ \\
\hline Especificaciones de entrada & Estar logueado en la aplicación. \\
\hline Criterios de aceptación & $\begin{array}{l}\text { Que el usuario pueda salir de su } \\
\text { cuenta seleccionando en el menú la } \\
\text { opción "cerrar sesión". }\end{array}$ \\
\hline Si cumple & \\
\hline
\end{tabular}

Tabla 23.

Prueba de aceptación 04 


\section{Conclusiones}

A través de la implementación de la metodología XP, llevando a cabo cada una de sus fases (planeación, diseño, codificación y pruebas), se desarrolló la aplicación de manera óptima y ágil.

La utilización de diagramas UML (Lenguaje Unificado de Modelado) permitió comprender la interacción entre el usuario y el sistema, así como la forma en que el sistema debe responder a las peticiones del usuario, presentando de manera visual el comportamiento esperado para iniciar la fase de programación.

Android Studio es de código libre y cuenta con una comunidad que ofrece sus conocimientos para ayudar a los programadores novatos, lo que significó un importante respaldo a la realización del proyecto para solucionar dudas al momento de realizar la programación.

La API (Application Programming Interface) Google Maps permitió integrar el mapa de la ciudad de Yopal y hacer el trazado mediante coordenadas para que el trayecto del autobús fuera visible en la aplicación $[6,7,8]$.

Por último, el inicio de sesión con la cuenta de Google de la aplicación facilita al usuario el proceso de registro e ingreso, lo cual conlleva a una mayor usabilidad 


\section{Referencias Bibliográficas}

1. "Supervisión de la tecnología: Las aplicaciones móviles alcanzan un nuevo hito", Itu.int, 2009. [Online]. Disponible en: https://www.itu.int/ net/itunews/issues/2009/06/04-es.aspx

2. K. Kendall and J. Kendall, Análisis y diseño de sistemas, 6th ed. México: Pearson, 2005, p. 20. [Online]. Disponible en: https:/ / books.google. com.co/s?id=SXD3OWcApcIC\&printsec $=$ frontcover\&dq=base + de + datos $\& \mathrm{hl}=\mathrm{es} \& \mathrm{sa}=\mathrm{X} \& \mathrm{sqi}=2 \&$ redir_esc $=\mathrm{y} \# \mathrm{v}=$ onepage\&q\&f$=$ false

3. “ Definición de casos de uso ", Ibm.com, (2017, Mar 03). [Online]. Disponible en: https://www.ibm.com/support/knowledgecenter/es/ SSWSR9_11.0.0/com.ibm.pim.dev.doc/pim_tsk_arc_definingusecases. html

4. "Diagramas de secuencia UML: Referencia", Msdn.microsoft.com, (2017, Mar 03). [Online]. Disponible en: https://msdn.microsoft.com/esco/library/dd409377.aspx

5. "Diagramas de actividades UML: Referencia", Msdn.microsoft.com, (2017, Mar 03). [Online]. Disponible en: https://msdn.microsoft.com/esco/library/dd409360.aspx

6. L. Ferrer Castellanos, K. González Insignares and L. Mendoza Vega, "La innovación como factor clave para mejorar la competitividad de las pymes en el departamento del Atlántico, Colombia", Dictamen Libre, no. 16, pp. 21-36, 2015.

7. P. app, "Probar tu app | Android Studio", Developer.android.com, (2017, Mar 16). [Online]. Disponible en: https://developer.android.com/ studio/test/index.html?hl=es-419

8. C. tarjetas, "Crear listas y tarjetas | Android Developers", Developer. android.com, (2017, Mar 16). [Online]. Disponible en: https://developer. android.com/training/material/lists-cards.html?hl=es 


\section{Bibliografía de consulta}

K. Beck, Una explicación de la programación extrema. Aceptar el cambio. Madrid: Pearson Educación, S.A., 2002.

C. Studio, "Conoce Android Studio | Android Studio", Developer.android. com, (2017, Feb 22). [Online]. Disponible en: https://developer.android. $\mathrm{com} /$ studio/intro/index.html?hl=es-419

About - Google Maps, Google.com, (2016, Feb 20). [Online]. Disponible en: https://www.google.com/maps/about/

Diseños | Android Developers", Developer.android.com, (2017, Abr 25. [Online]. Disponible en: https://developer.android.com/guide/topics/ ui/ declaring-layout.html?hl=es-419

Firebase, Firebase, (2016, Abr 16). [Online]. Disponible en: https:/ firebase. google.com/?hl=es-419

GOOGLE PLAY STORE, Google, (2016, Mar 02). [Online]. Disponible en internet: https:/ / play.google.com/about/developer-content-policy.html 


\section{Este artículo se cita}

P. Galindo., y M. Suárez, "Diseño e Implementación de una Aplicación Móvil Android para el Seguimiento de Rutas de Transporte Urbano en el Municipio de Yopal”, Investigación e Innovación en Ingenierias, vol. 5, nº 2, pp. 138-173, 2017 